\title{
Transición Para La Escolarización Del Preescolar A La Primaria Matemáticas Y Educación Física; Una Revisión Sistemática
}

\author{
Isaac Alejandro Aquino-Davila \\ Rigoberto Marín-Uribe \\ Facultad de Ciencias de la Cultura Física, Universidad Autónoma de \\ Chihuahua, México \\ Isabel Guzmán-Ibarra \\ Facultad de Filosofía y Letras, Universidad Autónoma de Chihuahua, \\ México
}

Doi:10.19044/esj.2021.v17n25p361

Submitted: 29 May 2021

Accepted: 08 July 2021

Published: 31 July 2021
Copyright 2021 Author(s)

Under Creative Commons BY-NC-ND 4.0 OPEN ACCESS

Cite As:

Aquino-Davila I.A., Marín-Uribe R. \& Guzmán-Ibarra I.(2021). Transición Para La Escolarización Del Preescolar A La Primaria Matemáticas Y Educación Física; Una Revisión Sistemática. European Scientific Journal, ESJ, 17(25), 361.

https://doi.org/10.19044/esj.2021.v17n25p361

\section{Resumen}

Se presenta una revisión sistemática del concepto de preparación para la escolarización (PPE), las dimensiones planteadas por el Fondo de las Naciones Unidas para la Infancia (UNICEF): escuelas, padres y "niños preparados”, enfocando esta última refiere al conjunto de competencias para que la infancia transite a la escuela primaria. El objeto de estudio busca realizar un análisis de las estrategias-instrumentos de evaluación, como parte de la PPE en ellos del campo pensamiento matemático y área educación física, en respuesta a la problematización en las formas de valorar este conjunto de competencias. El problema está referido a la forma de evaluar el preescolar en México. Para alcanzar el objetivo del estudio se utilizó la observación y el instrumento Escala para Evaluar Artículos Científicos en Ciencias Sociales y Humanas que examina, critica y sintetiza literatura representativa sobre un tema de forma integrada, de tal manera que permita comparar los hallazgos y generar nuevas perspectivas. Los artículos fueron consultados en los metabuscadores de SCOPUS, ELSEIVIER y SCIELO, en un periodo del 2017 
al 2021, filtrando 20 artículos para su revisión rigurosa. Se concluye que los instrumentos y formas evaluativas no solo pueden realizarse por psicometría, sino también en aportes que contribuyan a la valoración preescolar para una transición armónica.

Palabras clave: Preparación Para La Escolarización, Transición Escolar, Campo Formativo Matemáticas, Área De Desarrollo Educación Física

\title{
Transition To Schooling From Preschool To Primary: A Systematic Review
}

\section{Isaac Alejandro Aquino-Davila Rigoberto Marin-Uribe}

Faculty of Physical Culture Sciences, Autonomous University of Chihuahua, Mexico

Isabel Guzman-Ibarra

Faculty of Philosophy and Literature, Autonomous University of Chihuahua, Mexico

\begin{abstract}
A systematic review of the concept of preparation for schooling (PPE) is presented in this study. The dimensions proposed by the United Nations Children's Fund (UNICEF), schools, parents, and "prepared children" is focused on the latter and on the set of competencies required for childhood transitions into primary school. This paper focuses on performing an analysis of the evaluation strategies, i.e., instruments, as part of the preparation of school (PPE) in the field of mathematical thinking and physical education and in response to the problematization in evaluating this set of competences. The problem is however related to the way of evaluating preschool in Mexico. To achieve the objective of the study, observation and the Scale for Evaluating Scientific Articles in Social and Human Sciences instrument were used, which examines, criticizes, and synthesizes representative literature on a topic in an integrated way. It is done in such a way as to compare the findings and generate new perspectives. The articles were consulted in the metasearch engines of SCOPUS, ELSEIVIER, and SCIELO within the period from 2017 to 2021 by filtering 20 articles for rigorous review. In conclusion, the instruments and evaluative forms cannot only be performed by psychometry, but it also contributes to the preschool assessment for a harmonic transition.
\end{abstract}

Keywords: Preparation For Schooling, School Transition, Mathematics Training Field, Physical Education Development Area 


\section{Introduction}

Actualmente la PPE de niños y niñas del nivel educativo de preescolar, está ocupando más espacios en el mundo. Con el propósito de analizar la preparación para la escolarización, en la transición de los niños y niñas de prescolar a primaria, como parte de una investigación doctoral, este estudio se ubica en el currículo de la educación preescolar en México, de manera particular en el campo formativo: pensamiento matemático, y en el área de desarrollo personal y social: educación física, del Modelo Educativo del año de 2017 (SEP, 2017).

A pesar de que aún existen problemas referentes a una definición clara del concepto de PPE y sus dimensiones, que aplicada a los centros educativos contribuya al mejoramiento del aprendizaje y desarrollo de los niños y niñas, el UNICEF describe tres dimensiones dentro de la preparación para la escolarización: Niños preparados, Escuelas preparadas y Familias preparadas (Ainscow, 2019; Rebello \& Limlingan, 2012).

En este artículo se abordará la dimensión de "niños preparados”, en la búsqueda de estrategias e instrumentos de evaluación de la PPE, como objeto de estudio. Se parte de un concepto de PPE de los niños y niñas preescolares. Originalmente el concepto de "preparación escolar" (readiness school) fue definido como: un conjunto de habilidades predeterminadas físicas, sociales y cognitivas que los niños debían tener para cumplir con los requerimientos que exigía la escuela (Arnold et al., 2007, p. 36).

En este artículo se parte del concepto de PPE en los niños y niñas, como el conjunto de competencias deseables en su perfil de egreso, para tener una transición armónica del preescolar a la primaria (Arrivillaga et al., 2016). El conocimiento en la vida y el conocimiento en la escuela regularmente son relegados a conocimientos simplificados, enlatados y digeridos, que generan procesos de poco aprendizaje, sobre todo, al ser separados de actividades dadas en situaciones reales, esto es, no se crean espacios de continuidad para el aprendizaje sistematizado, orientado a cambiar las formas de enseñanza en la escuela y a mejorar la formación en la transición escolar, ya que la capacidad de aprender es evolutiva (Valderrama et al., 2019).

El currículo de la educación básica en México, que incluye a la educación preescolar, se conforma por tres componentes curriculares: cuatro ámbitos, tres campos formativos y tres áreas de desarrollo personal y social (SEP, 2017), como se observa en la Figura 1. 


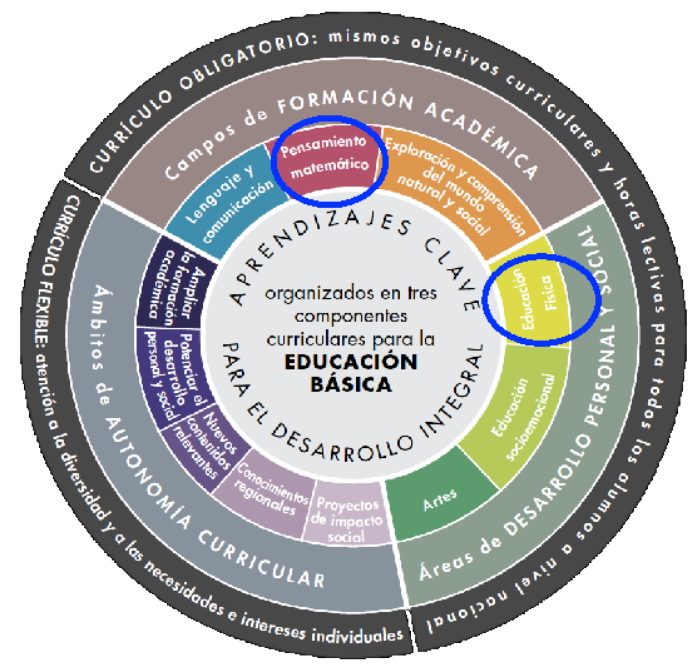

Figura 1. Aprendizajes clave para el desarrollo integral. Fuente: SEP (2017, p.25)

En este escrito, como parte del análisis, se revisan algunos documentos científicos relacionados con los y las preescolares en su transición a primaria, en el campo formativo del pensamiento matemático y en el área de desarrollo personal y social en educación física, atendiendo a la problematización sobre las diversas formas de valorar este conjunto de competencias.

El problema se focaliza en las prácticas evaluativas en preescolar donde, la tendencia en los estudios revisados, permite aventurar algunas reflexiones, entre otras, el hecho de asumir un nivel de desarrollo, similar en niños y niñas, como una condición para un tránsito del preescolar a la primaria, armónico, fácil y sin contratiempos, dando la impresión de homogeneidad en las condiciones institucionales, las normas, los métodos, los contenidos y los procesos de enseñanza-aprendizaje de la escuela primaria, pensar que son situaciones preestablecidas e inamovibles y validadas por la sociedad, de tal manera que para que la transición a la escuela sea exitosa, los niños y niñas, deberán ceñirse a condiciones de la escuela (Urbina, 2020).

Por lo anterior, se toma como punto de partida la valorización de la preparación escolar preguntándonos ¿Cuáles son las formas empleadas para evaluar la PPE, de manera específica, las estrategias para detectar el desarrollo psicomotor y matemático en los niños y niñas preescolares?

\section{Metodología}

Este estudio documental busca la identificación y el análisis de publicaciones sobre la "preparación para la escolarización”, a partir de la descripción de los procesos e interpretación crítica de la diversidad conceptual. Para alcanzar este objetivo, se asumió el método de la revisión integradora de la literatura trabajado por López et al. (2019) en el instrumento: 
"Escala para Evaluar Artículos Científicos en Ciencias Sociales y Humanas" (EACSH), a fin de generar nuevas perspectivas sobre el tema que proporcionen una comprensión más completa para su implementación en la práctica. A través de la consulta de investigaciones y publicaciones científicas originales, la revisión se realizó en las bases de datos de SCOPUS, ELSEVIER Y SCIELO. Los criterios adoptados para la selección de los artículos se muestran en la Tabla 1.

\begin{tabular}{|c|} 
Tabla 1. Criterios de inclusión \\
\hline Criterios de inclusión: \\
\hline Artículos originales \\
\hline Área temporal de publicación 2017 al 2021 \\
\hline Encontrados en: ELSEVIER, SCIELO y SCOPUS \\
\hline Artículos de Investigaciones cualitativas o mixtas \\
\hline Sinónimos de las palabras clave de búsqueda en inglés y español \\
\hline Resumen congruente a la dimensión del objeto de estudio \\
\hline Artículos no replicados \\
\hline
\end{tabular}

El periodo al que se acota la búsqueda de documentos es el comprendido entre los años de 2017 a 2021. Esto se justifica atendiendo a la temporalidad del modelo educativo de educación básica en México que surge en 2017 y que actualmente se encuentra vigente.

Los descriptores, en los criterios de inclusión, fueron referentes a las palabras clave:

- Preparación para la escolarización (readiness school)

- Transición preescolar (border crossing)

- Campo formativo matemáticas (mathematics)

- Área de desarrollo educación física (physical education)

Dichos descriptores fueron buscados tanto en español como inglés, asimismo, se utilizaron operadores de proximidad: sintácticos y semánticos, (NOT OR AND , ; . - + / “”) como se muestran en la Figura 2.

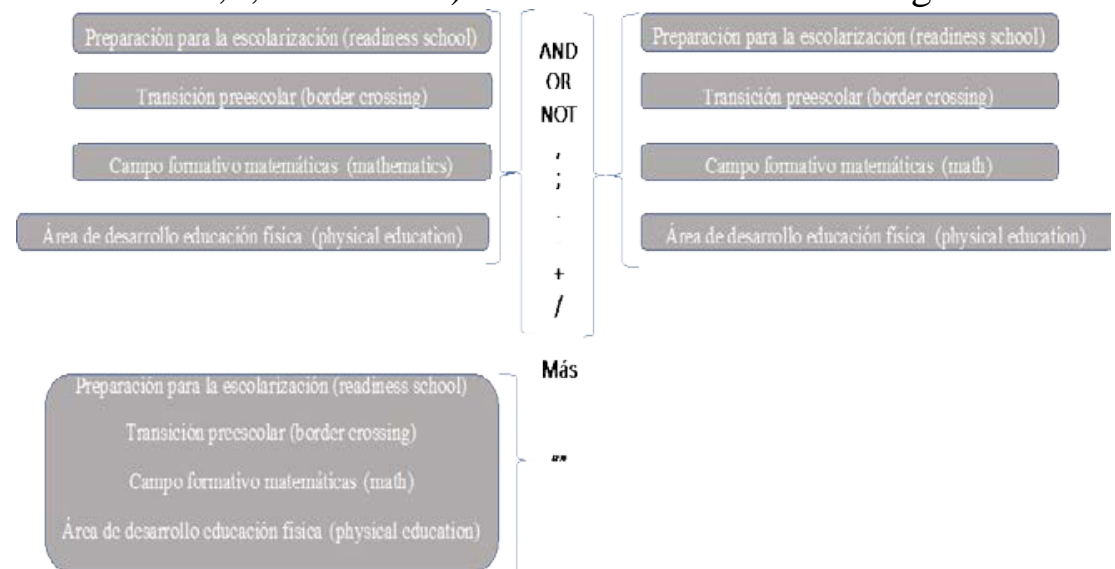

Figura 2. Operadores boléanos, sintácticos y dinámicos 
Los criterios de exclusión fueron las publicaciones que no cumplieron con los criterios de inclusión establecidos en este escrito, así como aquellos artículos que abordaron otra temática sobre la preparación para la escolarización diferentes al objeto de estudio.

En la primera etapa se encontraron 1255 documentos, los cuales fueron cribados empleando los primeros 3 criterios establecidos (Tabla 1), quedando un total de 414 artículos.

En la segunda etapa, aplicando los siguientes 3 criterios de inclusión, la mayoría de los artículos fueron rechazados por no ser afines al objeto de estudio, quedando finalmente 25 artículos, de los cuales, al aplicar el último criterio, se desecharon 5 por ser replicados en los metabuscadores, al eliminarlos, los 20 artículos restantes fueron sometidos a un análisis minucioso crítico y exhaustivo.

Esto permitió identificar los diferentes términos relacionados con la PPE. Por otra parte, se procedió a organizar un diagrama conceptual, a partir del cual se produjo la redacción de esta reflexión, que busca realizar un análisis sobre la transición de la PPE en los niños y niñas de preescolar a primaria en el campo formativo del pensamiento matemático y del área de desarrollo personal y social en educación física, atendiendo a la problemática de estudiar las diversas formas de valorar la PPE. Los artículos publicados en los tres metabuscadores utilizados y el flujo de la selección sistemática se muestran en el Figura 3.

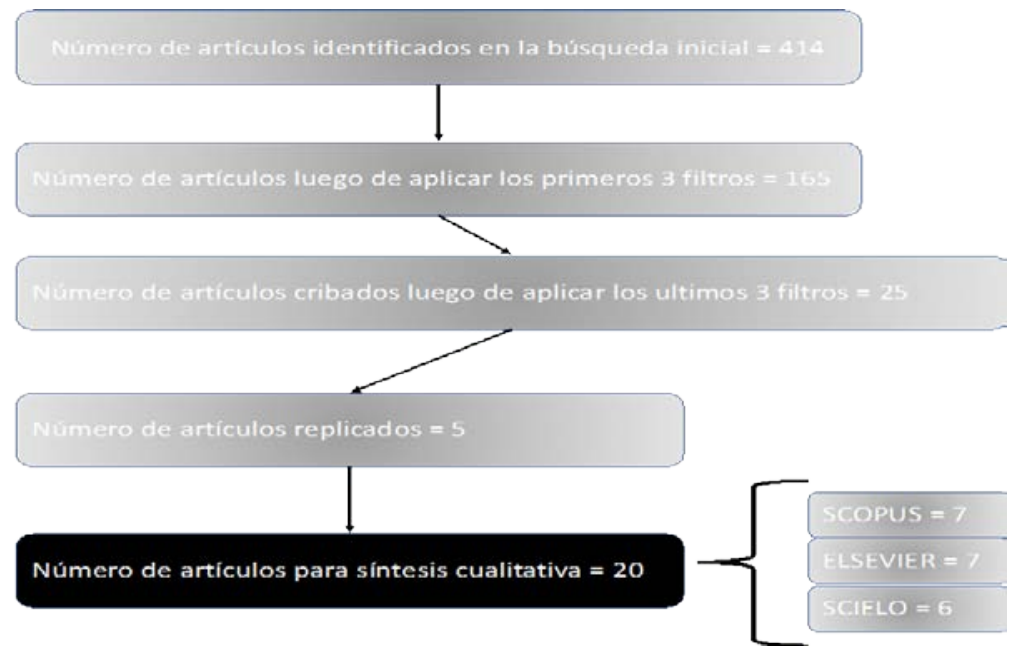

Figura 3. Flujo de la revisión sistemática para selección de artículos

\section{Resultados}

La revisión bibliográfica evidencia una amplia diversidad teórica en torno al proceso de PPE, ello implica la necesidad de mejorar la calidad educativa de la PPE de los niños y niñas mediante estrategias e instrumentos confiables y válidos, a fin de valorar de manera pertinente la educación de los 
niños y niñas para una transición armónica de los infantes del preescolar a la primaria (Arrivillaga et al., 2016; Bojorque et al., 2019; Suga et al., 2021).

En este tenor, con la intervención oportuna que prevenga trastornos del desarrollo, la ciencia ha resaltado la importancia del desarrollo infantil temprano, relacionado con los factores que identifican los riesgos de déficit del desarrollo psicomotor en la infancia. La trascendencia de conocer los factores de riesgo estriba en identificar aquellos niños que requieren de una intervención más dirigida que favorezca la expresión de su potencial (de Villiers et al., 2021; Reséndiz, 2020; Gamboa et al., 2018).

Por otra parte, se considera pertinente construir e implementar instrumentos de evaluación con el propósito de cuantificar y cualificar la preparación para la escolarización de los niños y niñas, que tome en cuenta aportes dentro del campo formativo del pensamiento matemático y área de desarrollo personal y social en educación física del preescolar a la primaria.

Con base en lo anterior, la interpretación de este proceso se enfoca a la búsqueda de elementos teóricos comunes que orienten el diseño de estrategias e instrumentos que lleven a evaluar de manera eficaz la PPE de los niños y niñas de preescolar y, en consecuencia, favorezcan su transición a la primaria.

A continuación, en las tablas 2, 3 y 4 se muestran aportaciones a la valoración relacionadas con la transición del preescolar a la primaria, cada una de las tablas maneja cuatro columnas, en la primera se presentan los autores como la muestra analizada en cada metabuscador; la segunda columna describe los instrumentos y herramientas usados por los autores, la tercera observa las categorías que ellos seleccionaron para sus investigaciones; finalmente, la cuarta columna muestra el análisis realizado por los autores

Tabla 2. Codificación de criterios con base en Elseiver

\begin{tabular}{|c|c|c|c|}
\hline Autor y año & $\begin{array}{c}\text { Instrumentos y/o } \\
\text { herramientas }\end{array}$ & Categorías & Análisis \\
\hline $\begin{array}{l}\text { de Villiers et } \\
\text { al. (2021) }\end{array}$ & $\begin{array}{c}\text { Herramienta } \\
\text { creada por los } \\
\text { autores para } \\
\text { evaluar lenguaje }\end{array}$ & $\begin{array}{l}\text { - Vocabulario } \\
\text { - Sintaxis } \\
\text { - Proceso }\end{array}$ & $\begin{array}{c}\text { Usan estrategias de } \\
\text { intervención en } 2 \text { tipos de } \\
\text { subpruebas, evaluan la } \\
\text { calidad de su programa. }\end{array}$ \\
\hline $\begin{array}{l}\text { Moreno et al. } \\
\text { (2020) }\end{array}$ & $\begin{array}{c}\text { Registros } \\
\text { conductuales }\end{array}$ & $\begin{array}{l}\text { - Ajuste escolar } \\
\text { - Problemas de } \\
\text { conducta } \\
\text { escolar } \\
\text { - Resultados } \\
\text { escolares }\end{array}$ & $\begin{array}{l}\text { Evaluar la eficacia del } \\
\text { programa Mindkinder } \\
\text { basado en atención plena } \\
\text { (mindfulness) sobre la } \\
\text { adaptación escolar. }\end{array}$ \\
\hline $\begin{array}{l}\text { Torrecilla y } \\
\text { Hernández } \\
\text { (2020) }\end{array}$ & $\begin{array}{l}\text { Aplica un análisis } \\
\text { multinivel de } \\
\text { cuatro niveles a } \\
\text { los datos del } \\
\text { Segundo Estudio } \\
\text { Regional } \\
\text { Comparativo y }\end{array}$ & $\begin{array}{l}\text { - Logros de } \\
\text { aprendizaje } \\
\text { - Factores } \\
\text { asociados }\end{array}$ & $\begin{array}{l}\text { Evalúan la participación de } \\
\text { los padres en la escuela y } \\
\text { como el proceso educativo } \\
\text { favorece el rendimiento } \\
\text { académico. }\end{array}$ \\
\hline
\end{tabular}




\begin{tabular}{|c|c|c|c|}
\hline & $\begin{array}{c}\text { Explicativo } \\
\text { (LLECE/UNESC } \\
\text { O, 2012) } \\
\end{array}$ & & \\
\hline $\begin{array}{l}\text { Fernández et } \\
\text { al. (2019) }\end{array}$ & $\begin{array}{c}\text { Test de Corsi } \\
\text { y } \\
\text { TEDI - MATH }\end{array}$ & $\begin{array}{c}\text { - Analiza la amplitud } \\
\text { de memoria } \\
\text { visoespacial } \\
\text { proporcionada para } \\
\text { el Diagnóstico de } \\
\text { las Competencias } \\
\text { Básicas. } \\
\end{array}$ & $\begin{array}{l}\text { Evalua e implementa un } \\
\text { programa virtual de } \\
\text { intervención. }\end{array}$ \\
\hline $\begin{array}{l}\text { Pastén et al. } \\
\text { (2018) }\end{array}$ & $\begin{array}{c}\text { Se evaluó por } \\
\text { medio de } \\
\text { problemas de } \\
\text { operaciones } \\
\text { lógicas y a través } \\
\text { de problemas con } \\
\text { enunciados orales } \\
\text { con y sin apoyo } \\
\text { visual. }\end{array}$ & $\begin{array}{l}\text { - Habilidades: } \\
\text { - Semánticas } \\
\text { - Morfosintácticas } \\
\text { - Conciencia } \\
\text { fonológica } \\
\text { - Memoria verbal } \\
\text { - Velocidad de } \\
\text { procesamiento } \\
\text { verbal. }\end{array}$ & $\begin{array}{c}\text { Evalúa la influencia } \\
\text { lingüística en resolución de } \\
\text { problemas matemáticos. }\end{array}$ \\
\hline $\begin{array}{l}\text { Utario et al. } \\
\text { (2018) }\end{array}$ & $\begin{array}{l}\text { Encuesta, registro } \\
\text { anecdótico e } \\
\text { inferencia. }\end{array}$ & $\begin{array}{c}\bullet \text { Examinan la } \\
\text { efectividad de una } \\
\text { aplicación de juego } \\
\text { móvil de Android } \\
\text { llamada Arbi Care } \\
\text { como un medio de } \\
\text { prevención. } \\
\end{array}$ & $\begin{array}{l}\text { Evalúan hábitos de higiene } \\
\text { asi como motricidad fina con } \\
\text { su intervención }\end{array}$ \\
\hline $\begin{array}{l}\text { Cahoon et al. } \\
\text { (2021) }\end{array}$ & $\begin{array}{c}\text { Análisis de } \\
\text { transición latente }\end{array}$ & $\begin{array}{c}\bullet \text { Evalúan tres } \\
\text { momentos } \\
\text { temporales en la } \\
\text { transición del } \\
\text { preescolar a la } \\
\text { primaria. }\end{array}$ & $\begin{array}{c}\text { Evalúan } 3 \text { tiempos lo que } \\
\text { permite una investigación de } \\
\text { diferentes vías de } \\
\text { aprendizaje de subgrupos } \\
\text { habilidades matemáticas en } \\
\text { la transición del preescolar a } \\
\text { la primaria. }\end{array}$ \\
\hline
\end{tabular}

En este sentido, Cahoon et al. (2021), de Villiers et al. (2021), Fernández et al. (2019), Moreno et al. (2020), Pastén et al. (2018), Torrecilla y Hernández (2020), y Utario et al. (2018) en sus estudios destacan la importancia de que, en la evaluación de los niños y niñas, su PPE, sus experiencias, su avance psicomotor y la función ejecutiva, logren desarrollarse plenamente, destacan a las habilidades matemáticas y el lenguaje como principales aspiraciones de la PPE en la búsqueda de que realicen una transición armónica del preescolar a la primaria.

Asimismo, Cahoon et al. (2021), Moreno et al. (2020), Torrecilla y Hernández (2020), Utario et al. (2018), dentro de las dimensiones de familias preparadas y escuelas preparadas; no solo destacan a la familia, también incluyen a sus docentes. De la misma manera, vinculadas a escuelas preparadas, analizan las necesidades socio-económicas que muestran grados 
extremos, en donde existen situaciones que develan necesidades de acceso a las TIC.

En el periodo de edad de los niños y niñas preescolares, debe considerarse su estado emocional, social y de salud, pues esto influye de forma determinante en la transición del hogar a la escuela. Deberá procurarse que la PPE de los niños y niñas se desarrolle en condiciones óptimas de salud, y de equilibrio emocional, sobre todo al enfrentar conflictos como: la muerte de un familiar, separación o divorcio de los padres, alguna enfermedad, por separación de la madre por motivo de viaje, nacimiento de un hermano y algunas otras situaciones en las que no conocemos el nivel de ansiedad en el niño, lo cual le resta confianza y seguridad en sí mismo (Moreno et al., 2020; Torrecilla \& Hernández, 2020; Fernández, 2019; Utario, 2018).

En la Tabla 3. podemos observar los documentos encontrados en el metabuscador Scopus.

Tabla 3. Codificación de criterios, con base en Scopus

\begin{tabular}{|c|c|c|c|}
\hline Autor y año & $\begin{array}{c}\text { Instrumentos y/o } \\
\text { herramientas }\end{array}$ & Categoría & Análisis \\
\hline $\begin{array}{l}\text { Herreras } \\
\text { (2017) }\end{array}$ & PISA 2012 & $\begin{array}{l}\text { - Matemáticas } \\
\text { - Fundamentales } \\
\text { - Desarrollo }\end{array}$ & $\begin{array}{l}\text { Analizar el riesgo de bajo } \\
\text { desempeño en función de } \\
\text { varias variables: asistencia a } \\
\text { la educación infantil, } \\
\text { estrategias de aprendizaje y } \\
\text { estrategias utilizadas por el } \\
\text { docente. }\end{array}$ \\
\hline $\begin{array}{l}\text { Suga et al. } \\
\text { (2021) }\end{array}$ & $\begin{array}{l}\text { Se analizaron } \\
\text { artículos de } \\
\text { intervención } \\
\text { originales sobre } \\
\text { actividad física } \\
\text { que utilizaron } \\
\text { métodos de } \\
\text { regresión, } \\
\text { publicados entre } \\
2000 \text { y } 2019 \text { en } \\
\text { inglés, portugués } \\
\text { y español. }\end{array}$ & $\begin{array}{c}\text { - Intervenciones } \\
\text { escolares } \\
\text { • Promover la } \\
\text { educación física } \\
\text { • Comportamientos } \\
\text { saludables }\end{array}$ & $\begin{array}{l}\text { Las intervenciones escolares } \\
\text { efectivas para los niños } \\
\text { pueden promover la } \\
\text { actividad física y los } \\
\text { comportamientos saludables. }\end{array}$ \\
\hline $\begin{array}{l}\text { Romero et al. } \\
\text { (2021) }\end{array}$ & $\begin{array}{c}\text { Utilizan la } \\
\text { regresión } \\
\text { jerárquica y el } \\
\text { análisis mediante } \\
\text { un modelo lineal } \\
\text { mixto. } \\
\text { f de Cohen y g de } \\
\text { Hedges. } \\
\text { Registro } \\
\text { anecdótico. }\end{array}$ & $\begin{array}{l}\text { - Programas de } \\
\text { intervención. } \\
\text { - La memoria de } \\
\text { trabajo } \\
\text { - La flexibilidad } \\
\text { cognitiva. }\end{array}$ & $\begin{array}{l}\text { Los resultados del estudio } \\
\text { muestran que el programa de } \\
\text { intervención tiene un } \\
\text { impacto significativo en } \\
\text { todas las variables de } \\
\text { función ejecutiva analizadas, } \\
\text { con grandes tamaños de } \\
\text { efecto. }\end{array}$ \\
\hline
\end{tabular}




\begin{tabular}{|c|c|c|c|}
\hline $\begin{array}{l}\text { Varghese et } \\
\text { al. (2019) }\end{array}$ & $\begin{array}{l}\text { Relaciones entre } \\
\text { maestros y } \\
\text { alumnos (TCR) }\end{array}$ & $\begin{array}{l}\text { El logro de } \\
\text { alfabetización } \\
\text { de los niños y } \\
\text { las } \\
\text { competencias } \\
\text { sociales. }\end{array}$ & $\begin{array}{l}\text { Analizan el vínculo entre } \\
\text { docente y los niños y niñas } \\
\text { en las experiencias y } \\
\text { aprendizajes en el aula } \\
\text { examinan las asociaciones } \\
\text { entre los TCR conflictivos y } \\
\text { cercanos }\end{array}$ \\
\hline $\begin{array}{l}\text { Pereira et al. } \\
\text { (2020) }\end{array}$ & $\begin{array}{l}\text { Escalas Mc } \\
\text { Carthy }\end{array}$ & $\begin{array}{c}\text { - Perfil de } \\
\text { desarrollo } \\
\text { psicomotor. } \\
\text { - Detectar posibles } \\
\text { desajustes. }\end{array}$ & $\begin{array}{c}\text { Se realizó un estudio de } \\
\text { cohorte prospectivo } \\
\text { longitudinal analítico } \\
\text { observacional con un diseño } \\
\text { de casos y controles, de } \\
\text { Aptitudes y Psicomotricidad } \\
\text { para niños. }\end{array}$ \\
\hline $\begin{array}{l}\text { Séve y Barray } \\
\text { (2020) }\end{array}$ & $\begin{array}{l}\text { Modelo analítico } \\
\text { observacional. } \\
\text { Las escalas Mc } \\
\text { Carthy de } \\
\text { aptitudes y } \\
\text { psicomotricidad } \\
\text { para niños. }\end{array}$ & $\begin{array}{c}\text { - Metodología de la } \\
\text { evaluación. } \\
\text { • Trastornos del } \\
\text { desarrollo. }\end{array}$ & $\begin{array}{l}\text { Se determinó el perfil de } \\
\text { desarrollo psicomotor de } \\
\text { niños con antecedentes de } \\
\text { prematuridad, a los } 4,5 \text { y } 6 \\
\text { años en la búsqueda de } \\
\text { evaluar de forma específica } \\
\text { cada circuito de la estructura } \\
\text { cognitiva modular. }\end{array}$ \\
\hline $\begin{array}{l}\text { Mercader et } \\
\text { al. (2017) }\end{array}$ & $\begin{array}{c}\text { Se analiza } \\
\text { longitudinalmente } \\
\text { la capacidad } \\
\text { predictiva de un } \\
\text { conjunto de } \\
\text { variables } \\
\text { motivacionales } \\
\text { sobre el } \\
\text { rendimiento } \\
\text { matemático. } \\
\end{array}$ & $\begin{array}{l}\bullet \text { Habilidades } \\
\text { matemáticas } \\
\text { - La dimensión } \\
\text { atribucional de } \\
\text { internalidad } \\
\text { positiva aportan } \\
\text { una contribución } \\
\text { significativa } \\
\text { adicional. }\end{array}$ & $\begin{array}{l}\text { En sus resultados muestran } \\
\text { un importante poder } \\
\text { predictivo sobre el } \\
\text { rendimiento matemático } \\
\text { posterior de la } \\
\text { autocompetencia percibida. } \\
\text { También la persistencia, la } \\
\text { actitud. }\end{array}$ \\
\hline
\end{tabular}

Herreras (2017), Mercader et al. (2017), Pereira et al. (2020), Romero et al. (2021), Séve y Barray (2020), Suga et al. (2021), Varghese et al. (2019), coinciden en que la evaluación de la PPE debe ser continua, no con el fin de asignar un número al niño o niña, sino más bien, para llevar a cabo una prevención oportuna, enfatizan en la necesidad de herramientas e instrumentos en la prevención y mejora continua en la educación.

Pereira et al. (2020), Romero et al. (2021), Suga et al. (2021), proponen y enfatizan en el empleo de situaciones o retos que los infantes relacionen con su vida cotidiana en la búsqueda de su desarrollo cardinal, tanto física como psíquica (psicomotor), de las habilidades que involucran los sentidos con la corporeidad exterosensorial y propiosensorial se interrelacionan y van religadas con el desarrollo del ser humano.

Aquellos niños en la vía de habilidades de números bajos, tienen una tasa de crecimiento más baja que los de vías más avanzadas, debido a la falta 
de comprensión de la cardinalidad. Herreras (2017), Séve y Barray (2020), Varghese et al. (2019), destacan la importancia del lenguaje y las habilidades de la memoria motora en el desarrollo de habilidades matemáticas.

Todos estos autores hacen énfasis en las experiencias de los niños y niñas durante su edad preescolar, Séve y Barray (2020) enfatizan la necesidad de intervenir-prevenir las necesidades educativas específicas, retrasos en el desarrollo y/o discapacidad en congruencia con Pereira et al. (2020) los programas de intervención que involucran psicomotricidad potencian las demás habilidades. Asimismo, aquellos aprendizajes logrados que involucren las emociones, se especulan, logran en los y las infantes un aprendizaje más significativo, aunado a que, si lográsemos hacer divertida la enseñanza, los párvulos adquieren gusto por el aprendizaje e, incluso, amor a la ciencia. Ya que están conociendo un mundo infinito.

Tabla 4. Codificación de criterios, con base en Scielo

\begin{tabular}{|c|c|c|c|}
\hline Autor y año & $\begin{array}{c}\text { Instrumentos y/o } \\
\text { herramientas }\end{array}$ & Categoría & Análisis \\
\hline $\begin{array}{l}\text { Gómez-León } \\
\text { (2020) }\end{array}$ & $\begin{array}{l}\text { Registro anecdótico } \\
\text { de las apraxias y } \\
\text { dispraxias } \\
\text { gestuales. }\end{array}$ & $\begin{array}{l}\text { • Entorno } \\
\text { favorable para } \\
\text { su óptimo } \\
\text { desarrollo. } \\
\text { Habilidades: } \\
\text { - Fisiológicas } \\
\text { - Cognitivas y } \\
\text { emocionales. }\end{array}$ & $\begin{array}{c}\text { Este trabajo pretende } \\
\text { sintetizar e integrar las } \\
\text { últimas aportaciones } \\
\text { empíricas sobre el desarrollo } \\
\text { de las habilidades creativas } \\
\text { en los niños con altas } \\
\text { capacidades durante la } \\
\text { infancia temprana. }\end{array}$ \\
\hline $\begin{array}{l}\text { Reséndiz } \\
\text { (2020) }\end{array}$ & $\begin{array}{l}\text { El análisis del } \\
\text { discurso escolar } \\
\text { respecto a la } \\
\text { enseñanza de la } \\
\text { noción de número } \\
\text { toma como } \\
\text { referencia a Piaget } \\
\text { y a Vygotsky. }\end{array}$ & $\begin{array}{l}\text { Procesos de } \\
\text { enseñanza- } \\
\text { aprendizaje. } \\
\text { - Diversas y } \\
\text { específicas } \\
\text { etapas } \\
\text { conforme a } \\
\text { sus } \\
\text { capacidades e } \\
\text { intelecto. }\end{array}$ & $\begin{array}{l}\text { analiza los procesos de } \\
\text { enseñanza-aprendizaje en } \\
\text { preescolar, donde se } \\
\text { encuentra inmersa la noción } \\
\text { de número, y, por otro lado, } \\
\text { el uso de las TIC para el } \\
\text { desarrollo de esta noción. }\end{array}$ \\
\hline Urbina (2020) & $\begin{array}{l}\text { Assessment and } \\
\text { Evaluation } \\
\text { Programming } \\
\text { System for Infants } \\
\text { and Children } \\
\text { (AEPS). } \\
\text { Consenso del uso } \\
\text { de prácticas de } \\
\text { transición de los } \\
\text { maestros. }\end{array}$ & $\begin{array}{l}\text { - Transición del } \\
\text { preescolar. } \\
\text { - Programa de } \\
\text { intervención. } \\
\text { - Practicas } \\
\text { educativas. }\end{array}$ & $\begin{array}{l}\text { Analiza los efectos a largo } \\
\text { plazo que puede tener la PPE } \\
\text { en niños de preescolar a } \\
\text { nivel académico y personal. }\end{array}$ \\
\hline $\begin{array}{l}\text { Bojorque et } \\
\text { al. (2019) }\end{array}$ & $\begin{array}{l}\text { habilidades } \\
\text { numéricas } \\
\text { tempranas y su }\end{array}$ & $\begin{array}{c}\text { - Habilidades } \\
\text { matemáticas. }\end{array}$ & $\begin{array}{l}\text { Los resultados indican que } \\
\text { existen diferencias } \\
\text { individuales en las }\end{array}$ \\
\hline
\end{tabular}




\begin{tabular}{|c|c|c|c|}
\hline & $\begin{array}{l}\text { enfoque espontáneo } \\
\text { en el número } \\
\text { (SFON). } \\
\text { Descripción } \\
\text { narrativa de la } \\
\text { situación } \\
\text { económica. }\end{array}$ & $\begin{array}{c}\text { • Nivel } \\
\text { socioeconómi } \\
\text { co. } \\
\text { • Competencias } \\
\text { numéricas. }\end{array}$ & $\begin{array}{l}\text { habilidades numéricas } \\
\text { tempranas y el SFON de los } \\
\text { niños ecuatorianos al iniciar } \\
\text { el jardín infantil. Además, el } \\
\text { nivel socioeconómico se } \\
\text { correlacionó positivamente } \\
\text { con las habilidades } \\
\text { numéricas tempranas, pero } \\
\text { no con el SFON. Al final se } \\
\text { discuten las implicaciones } \\
\text { teóricas, metodológicas y } \\
\text { prácticas del estudio. }\end{array}$ \\
\hline $\begin{array}{c}\text { Gamboa et al. } \\
\text { (2018) }\end{array}$ & $\begin{array}{c}\text { Identifica las } \\
\text { concepciones } \\
\text { orientadoras de las } \\
\text { prácticas corporales } \\
\text { con un carácter } \\
\text { innovador en un } \\
\text { estudio. }\end{array}$ & $\begin{array}{l}\text { - Innovación y } \\
\text { creatividad: } \\
\text { - De los niños } \\
\text { - De los docentes }\end{array}$ & $\begin{array}{l}\text { Los hallazgos relevan como } \\
\text { ejes centrales de la } \\
\text { innovación: su renovación, } \\
\text { contextualización social, } \\
\text { pensamiento renovador y } \\
\text { creativo del educador, } \\
\text { libertad de exploración por } \\
\text { parte de los niños y las niñas. }\end{array}$ \\
\hline $\begin{array}{l}\text { Bolaños et al. } \\
\text { (2018) }\end{array}$ & $\begin{array}{l}\text { Analizó la eficacia } \\
\text { de un programa de } \\
\text { intervención para } \\
\text { mejorar la PPE. }\end{array}$ & $\begin{array}{c}\text { - Comprensión } \\
\text { de las letras } \\
\text { minúsculas } \\
\text { del alfabeto } \\
\text { - Estudio de tipo } \\
\text { cualitativo }\end{array}$ & $\begin{array}{l}\text { Las investigaciones muestran } \\
\text { que las intervenciones psico- } \\
\text { educativas mejoran las } \\
\text { habilidades en los niños. }\end{array}$ \\
\hline
\end{tabular}

Bojorque et al. (2019), Bolaños et al. (2018), Gamboa et al. (2018), Gómez (2020), Reséndiz (2020), y Urbina (2020) coinciden en la necesidad de evaluar buscando como objetivo una transición armónica en la PPE, de manera continua y retroalimentada en la calidad, prevención y regularización de los niños y niñas; enfatizan en que desarrollar las habilidades visomotoras es esencial en la construcción del pensamiento complejo para que el niño adquiera la noción de conjunto y sub conjunto para darse cuenta que son infinitos en la naturaleza. El cálculo de velocidad y distancia lo desarrolla el infante de manera empírica: al correr, lanzar, brincar..., al combinarlas con el aprendizaje mediante la experiencia y repetición de los números, usando un pensamiento complejo en juegos y bailes más reglados comienza la noción del tiempo en números, la cual ya tenía de manera experiencial del día/noche.

Algunos autores como Bolaños et al. (2018) y Gamboa et al. (2018) sugieren que las relaciones entre un número limitado de variables se aplican a todos los alumnos de la misma manera, sin discriminar ni hacer la educación atractiva, por lo anterior, se considera que es necesario tomar aquellos referentes, construcciones y reflexiones pedagógicas que por años se han perfilado como el ideal, pero que en las dinámicas reales aún no logran impactar en una educación pertinente, oportuna, flexible, continua, preventiva 
y complementaria es primordial una transformación que verdaderamente logre cambiar.

Bojorque et al. (2019), Gómez (2020), y Urbina (2020) coinciden en un enfoque centrado en la persona con una transición latente rica en experiencias, habituar a los párvulos a buscar soluciones, experiencia individual y la percepción sensorial, en el descubrimiento de sus habilidades, los mecanismos psíquicos del ser que diera cuenta de la conexión entre las sensaciones físicas, los juicios y razonamientos.

Replicar cosas simples como preparar el desayuno en la escuela implica que los infantes adquieran nociones de cantidad, al servir comida o bebida, así como, medida al acomodar platos y/o sillas (Gamboa et al., 2018; Bolaños et al., 2018).

Reséndiz (2020) cuestiona la enseñanza de la noción de número, toma como referencia a Piaget y a Vygotsky, sin embargo, algunos juegos y estrategias son rescatables, como, por ejemplo, la repetición motora y contable. Bojorque et al. (2019) y Bolaños et al. (2018) muestran como la repetición invade la memoria y la curiosidad del niño y la niña.

Por otra parte, Reséndiz et al. (2020) refieren que la transición de un grado escolar a otro requiere de un proceso de adaptación, que apoye las experiencias para adaptarse a un terreno desconocido, las aulas o instalaciones, sus compañeros también, pero serán las nuevas vivencias de retos sociales, académicos y personales los que harán la diferencia.

Al recolectar los artículos analizados, en los tres meta buscadores, todos los autores proponen regularización, prevención y propuestas afines, así como la necesidad de crear psicometría con el fin de buscar que la PPE sea suficiente para que los niños y niñas, se desenvuelvan adecuadamente en su transición a la primaria.

Bojorque et al. (2019), Bolaños et al. (2018), Cahoon et al. (2021), De Villiers et al. (2021), Fernández et al. (2019), Gamboa et al. (2018), Gómez (2020), Herreras (2017), Mercader et al. (2017), Moreno et al. (2020), Pastén et al. (2018), Pereira et al. (2020), Reséndiz (2020), Romero et al. (2021), Torrecilla y Hernández (2020), Séve y Barray (2020), Suga et al. (2021), Urbina (2020), Utario et al. (2018), Varghese et al. (2019), hacen énfasis no solo en la dimensión de niños preparados si no, también la dimensión de escuelas preparadas, en la búsqueda de una educación de calidad y aprendizajes significativos.

\section{Discusión}

Durante la transición escolar, el niño presenta un grado de temor debido a que no tiene conocimiento de lo que va a encontrar o sobre el ambiente social al que está a punto de ingresar. El comenzar la escuela es algo nuevo totalmente, ya que dentro de este nivel escolar el niño deberá obedecer 
a otros adultos que están fuera de su círculo familiar, así como convivir con otros niños de su misma edad (Moreno et al., 2020; Varghese et al., 2019).

Se debe tomar en cuenta el estado emocional, social y de salud del niño como parte de su PPE, ya que esto influirá de manera determinante por lo que se debe procurar que el niño esté en condiciones óptimas de salud y de equilibrio emocional, por ello es conveniente que cuando está atravesando por situaciones difíciles, en las que no conocemos el nivel de ansiedad en el niño se encuentra, cuidemos aquello que le resta confianza y seguridad en sí mismo (Moreno et al., 2020; Varghese et al., 2019).

Si el niño se encuentra en "óptimas condiciones” para poder ingresar a la educación primaria, los padres serán los encargados de hablar con el niño para que este conozca del nuevo cambio que va vivir y el ambiente que se puede llegar a encontrar (Suga et al., 2021; Herreras, 2017).

La PPE de los niños y niñas, en la transición del preescolar a la primaria, o el someterse a ciertos ritos en el tránsito propio de su cultura, representa desafíos y oportunidades para el aprendizaje y el crecimiento en múltiples niveles. La transición de preescolar a primaria es la más importante, ya que la experiencia que se obtenga de esta, sea positiva o negativa, afectará en la escolaridad futura, la que ocasionará un sentimiento de éxito o de fracaso escolar (Romero et al., 2021; Pereira et al., 2020).

López y de Pro-Bueno (2020) destacan que la educación preescolar tendrá como objetivos especiales el promover y estimular el desarrollo físico, afectivo y espiritual del niño, su integración social, su percepción sensible y el aprestamiento para las actividades escolares, en acción coordinada con los padres y la comunidad, por lo que sus escritos pretenden impactar en las tres dimensiones planteadas por el UNICEF (Ainscow, 2019).

En las tres dimensiones planteadas por el UNICEF (Ainscow, 2019; Rebello \& Limlingan, 2012) las actividades físicas y matemáticas son menester de la vida cotidiana, que involucran al niño y la sociedad, son una necesidad prioritaria de un trabajo multidisciplinar entre escuelas, familias y niños.

Riascos et al. (2020), Torrecilla y Hernández (2020) consideran que la transición se basa en las acciones que favorecen el paso de un nivel a otro, siendo el salto del preescolar a la primaria el gran reto pues involucra a toda la comunidad educativa, Esto porporciona una experiencia significativa que ya está en marcha en los preescolares cuando se implementa el desarrollo integral, donde el juego, el canto, la exploración del medio, el arte, la literatura, la expresión corporal, las mesas redondas, el trabajo colaborativo, experimentar, las dimensiones del desarrollo y la evaluación integral del ser, son fundamentales en la PPE que los niños y niñas requieren en su transición. En congruencia con las concepciones de Riascos et al. (2020) y Fernández et al. (2019) el concepto de transición educativa hace referencia al cambio que 
efectúan los niños desde una fase educativa a otra; es en estos cambios donde los pequeños se enfrentan a desafíos relativos a las relaciones sociales, el estilo de enseñanza, el ambiente, el espacio, el tiempo, los contextos de aprendizaje y el aprendizaje mismo, lo cual hace que este proceso resulte ser intenso y con demandas crecientes. Cuando los estudiantes del grado preescolar realizan la transición educativa a la primaria los procesos escolares cambian y se encuentran, con diferentes metodologías, ambientes y dinámicas, lo cual hace que este tránsito se vea fracturado. Generando la necesidad de articular los procesos para que se dé una transición armónica.

Asimismo, Riascos et al. (2020) y Pastén et al. (2018) plantean que para que la transición sea armónica es de vital importancia el acompañamiento familiar en el proceso de formación integral de los estudiantes. La participación de la comunidad educativa en este proceso contribuye notablemente al generar interés en la adquisición de los aprendizajes significativos de los estudiantes, este acompañamiento debe ser continuo, los estudiantes necesitan sentir el apoyo de la familia y el de la institución educativa, ya que la efectividad en los procesos educativos depende en gran manera de estos.

Por lo anterior, se considera establecer que las transiciones implican un cambio, en el cual ocurren procesos de continuidad, progresión y diferenciación, en los cuales pudiera haber un encadenamiento cada uno de ellos juega un rol en el paso al nuevo estado. Uno da estabilidad, otro complejiza lo alcanzado y el tercero ofrece el cambio de lugar, se da en la simultaneidad entre uno y otro ambiente. La PPE para el paso entre ambientes diferentes, se da en el tránsito del preescolar a la educación básica, en este momento se debe establecer un proceso de continuidad progresiva que beneficie a los estudiantes en la interacción, socialización y adaptación al siguiente nivel educativo, favoreciendo la transición en esta etapa escolar (Mercader et al., 2017; Utario et al., 2018).

De acuerdo con lo anterior, es necesario generar procesos que contribuyan al fortalecimiento de la interacción social, comunicativa, asertiva, ideológica y de compartir del trabajo colaborativo como parte de la PPE, que permita la adaptación de los estudiantes al nuevo entorno educativo para potenciar así un ambiente óptimo para la transición armónica. Asimismo, para dar continuidad al proceso entre el último grado preescolar y primero de primaria, se debe implementar una estrategia didáctica que cuente con la participación e integración de la comunidad educativa en aras de construir un puente que facilite la integración y adaptación de los estudiantes a las nuevas dinámicas escolares.

Gómez y Gómez (2021) y Moreno et al. (2020) plantean en sus resultados que para muchos niños y niñas el paso por el preescolar se convierte en un hermoso y mágico recuerdo de la infancia, es allí donde aprenden a 
socializar, a compartir, se integran y experimentan con los compañeros y profesores. En este momento se evidencia la importancia de buscar estrategias que beneficien la transición escolar, para que los estudiantes disfruten de este proceso y creen hermosos recuerdos de la experiencia vivida en esta etapa de sus vidas.

En correspondencia con las concepciones de Gómez y Gómez (2021) y Pereira et al. (2020), comprender cómo las niñas y niños pequeños están mejor preparados para ingresar y tener éxito en la escuela primaria ha llegado a ser una prioridad entre los padres, educadores, legisladores e investigadores. Es así como la transición entre los grados se ha vuelto una necesidad en la generación de espacios sin vacíos en el currículo y pedagogía. La transición armónica se basa en acciones que favorecen el paso de un nivel a otro, en el contexto educativo con base en las estrategias didácticas implementadas para que se creen vínculos de comunicación en los cuales se compartan las metodologías de enseñanza y aprendizaje, para dar continuidad y seguimiento en los procesos educativos (Romero et al., 2021).

\section{Conclusiones}

Se concluye, a partir de los elementos comunes encontrados en las publicaciones revisadas, en la necesidad de construir e implementar instrumentos y estrategias evaluativas con el propósito de cuantificar y cualificar la preparación para la escolarización de los niños y niñas de preescolar en su transición a la primaria. Esto deberá tomar en cuenta aportes de prevención e intervención dentro del campo formativo del pensamiento matemático y área de desarrollo personal social en educación física.

Se considera oportuno resaltar que el perfil de preescolar es el principal componente para avanzar en la construcción de instrumentos y estrategias de evaluación lleven a valorar la PPE de los niños de preescolar. En dicho perfil deberán aparecer los campos y áreas del modelo educativo que opera la educación básica, así como los organizadores curriculares y aprendizajes esperados articulados a fin de facilitar la evaluación integral de los niños y niñas de preescolar en su tránsito a la primaria.

Con este propósito, se busca clarificar la interpretación que se les confiere a los procesos de transición de un nivel de enseñanza a otro y se concluye que las prácticas evaluativas que establecen los centros siguen, en la mayoría de los casos, planes que el mismo centro ha elaborado; asimismo, entre las dificultades, se resaltan la falta de autonomía y la diferencia de niveles de lectura y escritura con los que se promociona el alumnado de un nivel a otro en la educación prescolar. 


\section{References:}

1. Ainscow, M. (2019). Años después de la Declaración de Salamanca de la UNESCO. Crear sistemas educativos inclusivos y equitativos. Documento de Discusión Preparado Para El Foro Internacional Sobre Inclusión y Equidad, 11-13.

2. Arnold, C., Bartlett, K., Gowani, S. y Merali, R. (2007). Is Everybody Ready? Readiness, transitions and continuity-Reflections and moving forward, Working Papers in Early Childhood Development 41, The Hague, The Netherlands: Bernard van Leer Foundation. P. 36.

3. Arrivillaga, C., Cuevasanta, D., Liz, M., Moreira, K., Schiappacasse, P., y Echeverría, A. V. (2016). Preparación para la escolarización: una revisión sistemática de estudios longitudinales. PSIENCIA. Revista Latinoamericana de Ciencia Psicológica, 8(1), 1-12.

DOI: http://dx.doi.org/10.5872/psiencia.v8i1.191

4. Bojorque, G., Torbeyns, J., Hoof, J. V., Nijlen, D. V., y Verschaffel, L. (2019). Influencia del nivel socioeconómico en el desarrollo de las competencias numéricas de los niños ecuatorianos de jardín infantil. Perfiles educativos, 41(166), 90-104.

5. Bolaños-Motta, J. I., Pérez-Rodríguez, M. A., y Casallas-Forero, E. (2018). Alfabetización Corporal. Una propuesta de aula desde la psicomotricidad. Estudios pedagógicos (Valdivia), 44(3), 23-34.

6. Cahoon, A., Gilmore, C., y Simms, V. (2021). Developmental pathways of early numerical skills during the preschool to school transition. Learning and Instruction, 75, 101484.

https://doi.org/10.1016/j.learninstruc.2021.101484

7. de Villiers, J., Iglesias, A., Golinkoff, R., Hirsh-Pasek, K., Wilson, M. S., y Nandakumar, R. (2021). Assessing dual language learners of Spanish and English: Development of the QUILS: ES. Revista de Logopedia, Foniatría y Audiología. Articles in press, Disponible online el 6 de Enero de 2021. DOI: 10.1016/j.rlfa.2020.11.001

8. Fernández-Abella, R., Peralbo-Uzquiano, M., Durán-Bouza, M., Brenlla-Blanco, J. C., y García-Fernández, M. (2019). Virtual Intervention Programme to Improve the Working Memory and Basic Mathematical Skills in Early Childhood Education. Revista de Psicodidáctica, 24(1), 17-23

9. Gamboa Jiménez, R. A., Jiménez Alvarado, G. A., Peña González, N. J., Gaete Navarro, C.F., y Aguilera Ubeda, D. J. (2018). Prácticas corporales e innovación en educación infantil (0-6 años): análisis crítico desde la mirada de expertos. Revista Brasileira de Ciências do Esporte, 40(3), 224-232.

10. Gómez-León, M. I. (2020). Bases psicobiológicas de la creatividad en los niños con altas capacidades. Psiquiatría Biológica, 27(1), 28-33. 
11. Gómez-Marí, I. y Gómez-Marí, P. (2021). Orientaciones pedagógicas: ¿cómo facilitar el proceso de transición del alumnado desde la etapa de educación Infantil a Primaria? Percepciones de familias y profesionales de la educación. REIDOCREA, 10(4), 1-14.

12. Herreras, E. B. (2017). Risk low math performance PISA 2012: Impact of assistance to Early Childhood Education and other possible cognitive variables. Acta de investigación psicológica, 7(1), 26062617.

13. López Portela, L. X., y de Pro-Bueno, A. (2020). Historia de la educación inicial en Colombia: démosle un giro a ese cuento. Actualidades Pedagógicas, 1(75), 131-156.

14. López, E. L., Tobón, S., y Hernández, L. G. J. (2019). Escala para evaluar artículos científicos en ciencias sociales y humanas-EACSH. REICE: Revista Iberoamericana Sobre Calidad, Eficacia Y Cambio En Educación, 17(4), 111-125.

15. Mercader, J., Presentación, M. J., Siegenthaler, R., Molinero, V., y Miranda, A. (2017). Motivation and mathematics performance: a longitudinal study in early educational stages. Revista de Psicodidáctica (English ed.), 22(2), 157-163.

16. Moreno-Gómez, A., Luna, P., y Cejudo, J. (2020). Promoting school success through mindfulness-based interventions in early childhood. Revista de Psicodidáctica, 25(2), 136-142.

17. Pastén, L. E., Taverner, R. M., y Fernández, A. Y. (2018). Conciencia fonológica y resolución de problemas matemáticos en educación infantil. Revista de Logopedia, Foniatría y Audiología, 38(2), 61-68.

18. Pereira-Cerro, A. V., Lanzarote-Fernández, M. D., Barbancho-Morant, M. M., y Padilla-Munoz, E. M. (2020, October). Evolución del desarrollo psicomotor en preescolares con antecedentes de prematuridad. Anales de Pediatría 93 (4), 228-235. Elsevier Doyma. https://doi.org/10.1016/j.anpedi.2019.10.003

19. Presa, T., Vásquez Echeverría, A., Mandl Ruiz, A. I., Méndez, E., y Climente, C. (2019). Las habilidades socioemocionales como facilitadoras de la transición en primer ciclo y su contribución con la disposición para la escolarización en lecto-escritura a partir de los resultados del INDI (Inventario de Desarrollo Infantil). http://repositorio.cfe.edu.uy/handle/123456789/1161

20. Rebello Britto, P. y Limlingan, M. (2012). School Readiness and Transition. A companion to the Child Friendly Schools Manual (pp. 128). UNICEF. 
21. Reséndiz-Balderas, E. (2020). Análisis del discurso y desarrollo de la noción de número en preescolar y el uso de las TIC. Ciencia UAT, 14(2), 72-86.

22. Riascos Ordoñez, N., Vargas Díaz, X., y Zambrano Trompeta, M. Y. (2020) Estrategia Didáctica para la Transición entre el último grado de Preescolar y el Primero de Básica Primaria. http://hdl.handle.net/11634/27543

23. Romero-López, M., Pichardo, M. C., Justicia-Arráez, A., y CanoGarcía, F. (2021). Effect of the EFE-P program on the improvement of executive functions in Early Childhood Education. Revista de Psicodidáctica, 26(1), 20-27.

24. Secretaría de Educación Pública (SEP). (2017). Modelo Educativo para la educación obligatoria. Autor.

25. Sève-Ferrieu, N. y Barray, V. (2020). Evaluación de la apraxia gestual y de la dispraxia en el marco de los trastornos neuropsicológicos. EMC-Kinesiterapia-Medicina Física, (41)2, 1-16.

26. Suga, A. C. M., da Silva, A. A. D. P., Brey, J. R., Guerra, P. H., y Rodriguez-Añez, C. R. (2021). Effects of interventions for promoting physical activity during recess in elementary schools: a systematic review. Jornal de Pediatria. En prensa: online 25 March 2021. https://doi.org/10.1016/j.jped.2021.02.005

27. Torrecilla, F. J. M. y Hernandez-Castilla, R. (2020). Does parental involvement matter in children's performance? A Latin American primary school study. Revista de Psicodidáctica, 25(1), 13-22.

28. Urbina-García, M. Á. (2020). Programa de Intervención para facilitar la Transición al Primer año de Primaria. Diálogos sobre educación. Temas actuales en investigación educativa, 11(21), 1-19. https://doi.org/10.32870/dse.v0i21.664

29. Utario, Y., Rustina, Y., Krianto, T., y Ayubi, D. (2018). Arbi Care application increases preschool children's hand-washing self-efficacy among preschool children. Enfermeria clinica, 28, 27-30.

30. Valderrama, C. R., Floody, P. D., Espinoza-Silva, M., y Mayorga, D. J. (2019). Comportamiento del Profesor, Intensidad y Tiempo Efectivo de las Clases de Educación Física en una escuela pública: Un acercamiento a la realidad. Retos: nuevas tendencias en educación física, deporte y recreación, 35, 160-163.

31. Varghese, C., Vernon-Feagans, L., y Bratsch-Hines, M. (2019). Associations between teacher-child relationships, children's literacy achievement, and social competencies for struggling and nonstruggling readers in early elementary school. Early Childhood Research Quarterly, 47, 124-133. 